\title{
An Extension of Seam Pucker Simulation to Manage Collisions
}

\author{
Shigeru Inui \\ National Institute of Materials and Chemical Research, 1-1, Higashi, Tsukuba, Ibaraki 305 Japan
}

\begin{abstract}
The seam pucker simulation was extended to be able to treat collisions. In case of seam pucker simulation, an element of one virtual fabric might not collide with the remote portion of another virtual fabric. The local search of collisions is enough for the purpose of seam pucker simulation. For the detection of collisions, "selection" which is one of the functions of a graphic library was utilized. Collisions were settled that the colliding elements were made to contact with each other. Two ways of collision management were proposed. One was the iterated use of "selection" and the other was the geometrical calculation. To utilize for the calculation of deformation, the force from one fabric to another through the contact point was calculated. A test simulation of seam pucker was executed. Another test simulation of an elastic cube wrapped with a fabric was also executed and the proposed collision management was verified.
\end{abstract}

\section{Introduction}

We presented a seam pucker simulation in the former report[1]. In the simulation, virtual fabric is divided into triangular elements. Internal force works on each apex of triangular elements and the energy owing to deformation caused by the force are calculated[2]. The internal force is calculated from the linear relationship of stress and strain about tensile, shear and bending. Every apex is moved according to the force to minimize the total energy. The minimal energy state determines the shape of virtual fabric.

The type of the problem treated here is as follows. Fig. 1 shows one of the results of the seam pucker simulation. In the simulation, the shape of the seam pucker sample composed of one strip of fabric was calculated. The center of the strip was regarded to be a seam and the length of that was shrunk as an initial condition of the simulation. However real seam pucker samples consist of two strips of fabrics. With our previous program, collisions between elements of virtual fabrics might frequently happen as shown in Fig.2. We had to improve the simulation program to process collisions. The collision problem can be divided

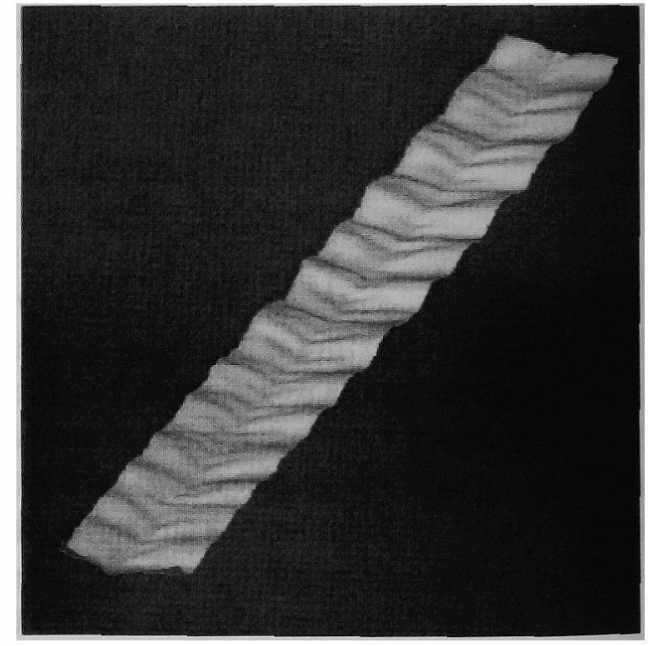

Fig. 1 A result of seam pucker simulation.

into two parts. One is the search of the elements which may collide with an element and the other is the calculation how an element collide with another one. In the former part, the search process may be global. For example, in case of the calculation of the shape of a curtain, an element may collide with the element located in remote position of the same fabric. On the other hand, in the simulation of seam pucker with two 




Fig. 2 Collision of fabrics in the seam pucker simulation.

strips of fabrics, only a restricted area can collide with an element in another fabric and an element in one fabric never collide with remote elements of another fabric. Fig. 3 illustrates this situation. In case of seam pucker, the global search is not necessary and we focused the calculation how the elements collide with.

\section{Experimental}

\subsection{Collision Detection}

We utilized "selection" [3] which is one of the functions of a graphic library for the purpose of the

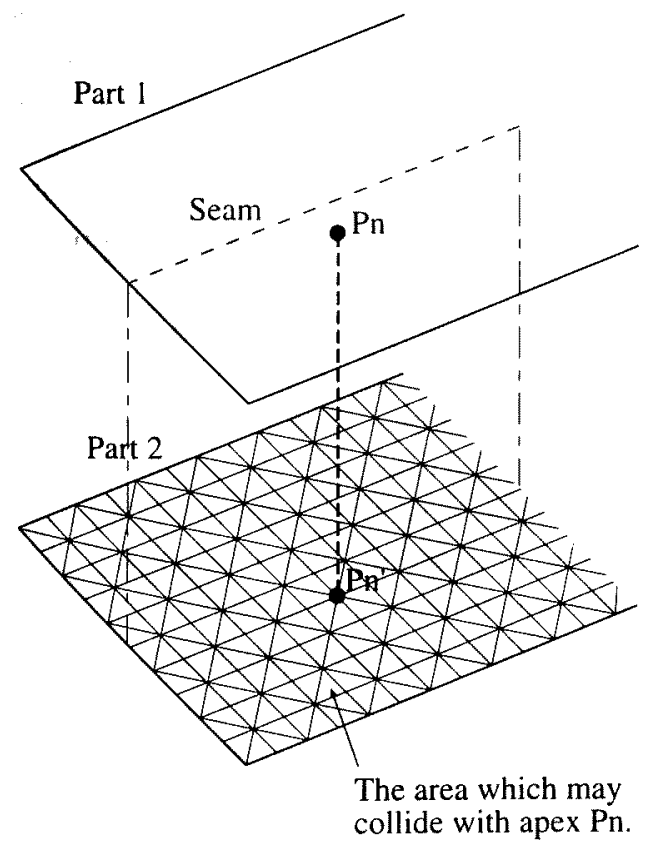

Fig. 3 Provable area of collision. detection of collisions between geometrical elements. "Selection" is a technique to detect geometrical elements that are included in a three-dimensional region. A viewing volume which is usually defined as a rectangular parallelepiped or a frustum is used for the three-dimensional region. Fig. 4 illustrates the function of "selection". Six planes of a viewing volume clip a three dimensional space and define a region of "selection". In addition to the planes of a viewing volume, another clipping planes can be added and the shape of the region for "selection" can be modified as shown in Fig.5. The graphic library which contains "selection" can be utilized in various kinds of computers. In case of the computers which have

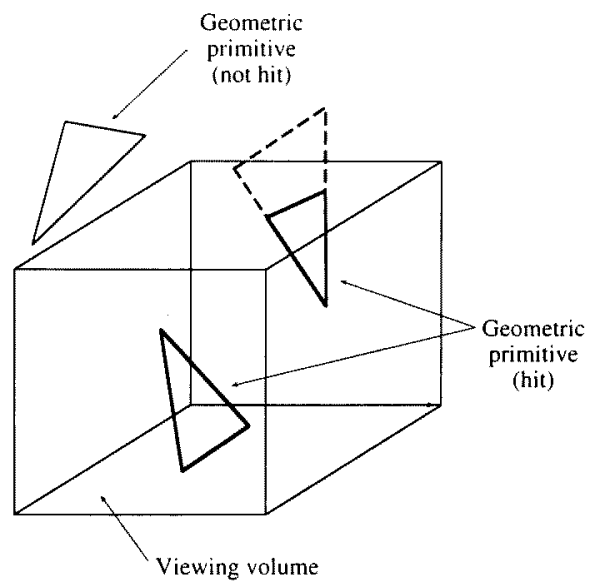

Fig. 4 Selection with viewing volume.

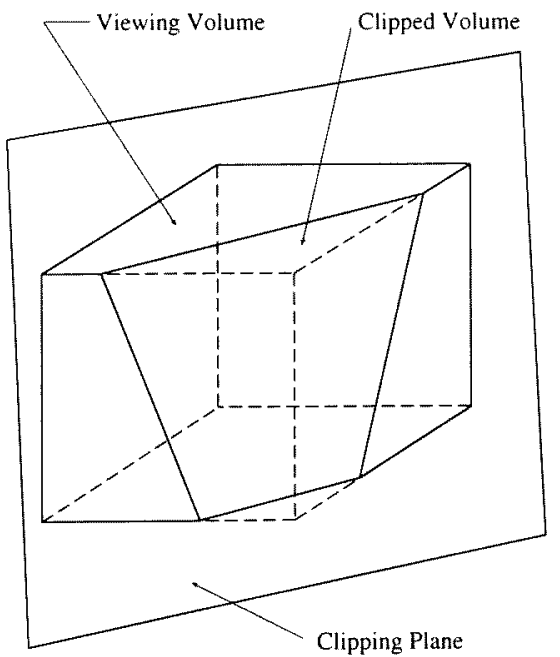

Fig. 5 Clipped viewing volume. 
hardware accelerator of geometrical calculation for graphics, "selection" is executed faster.

\subsection{Collision management}

Fig. 6 shows the status when an apex is displaced according to the force acts on it. In the figure, the apex is included in four triangular elements. The bold allow line shows the displacement of the apex. The start point of the allow line is the position of the apex before displacement and the end point is that after displacement. The dashed lines show the sides of triangular elements after the apex moves. We took the tetrahedron which was made up with a triangular element before displacement of the apex and the bold allow line as the region for "selection". Then the triangular elements which collide with the tetrahedron are picked up. This check process is executed for every triangular elements that include the apex. After that, how the triangular elements collide with the tetrahedron is examined for all the tetrahedrons containing the displaced apex. There are two ways of the examination. One is the way which uses only "selection". Fig. 7 shows the procedure. In the figure, $P_{1}, P_{2}$ and $P_{3}$ are apexes of a triangular element and $P_{3}$ is the position of the apex before it is displaced and $\mathrm{P}_{4}$ is that after it is displaced. In the first step, if some triangular elements collide with the tetrahedron, the tetrahedron vertex $\mathrm{P}_{4}$ is moved to $\mathrm{P}_{4}$ ' which is the middle of $\mathrm{P}_{3} \mathrm{P}_{4}$. In the next step, $\mathrm{P}_{4}{ }^{\prime}$ is moved to $\mathrm{P}_{4}{ }^{\prime \prime}$. If the new tetrahedron with $\mathrm{P}_{4}$ ' collides with any triangular element, $\mathrm{P}_{4}{ }^{\prime \prime}$ is the middle of $\mathrm{P}_{3} \mathrm{P}_{4}$ ' and if it does not collide, $\mathrm{P}_{4}$ " is the middle of $\mathrm{P}_{4}{ }^{\prime} \mathrm{P}_{4}$. After a number of iterations, $\mathrm{P}_{3}$ is converged upon the point which is a vertex of the tetrahedron that does not collide with any triangular element and is closest to $\mathrm{P}_{4}$.

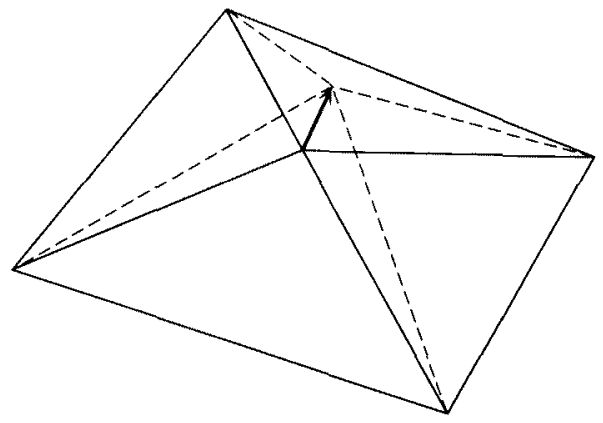

Fig. 6 Movement of an apex for energy minimization.

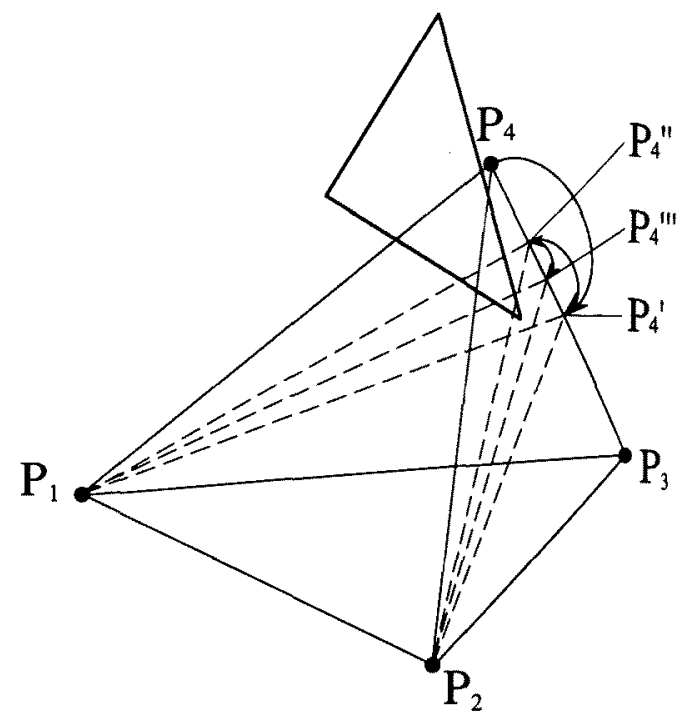

Fig. 7 Iterated use of "selection".

The number of the iterations was determined beforehand according to the required precision. Another way of the examination is geometrical one. Three collision modes are considered as illustrated in Fig. 8. In collision mode 1, an apex is included in the tetrahedron. In this mode, $P_{4}$ is pulled back on the side $\mathrm{P}_{3} \mathrm{P}_{4}$ from $\mathrm{P}_{4}$ to $\mathrm{P}_{4}$ ' where the apex of colliding triangular element contacts with moved triangular element $\mathrm{P}_{1} \mathrm{P}_{2}$ $\mathrm{P}_{4}$ 'as shown in Fig.8. In collision mode 2, a side of the colliding triangular element pierces the tetrahedron. In this mode, $\mathrm{P}_{4}$ is pulled back to $\mathrm{P}_{4}{ }^{\prime}$ where the side of the colliding triangular element contacts with the side $\mathrm{P}_{1} \mathrm{P}_{4}^{\prime}$ or $\mathrm{P}_{2} \mathrm{P}_{4}$. In collision mode 3 , all the apexes and sides are out of the tetrahedron but the plane of the triangular element cuts the tetrahedron. In this mode, $\mathrm{P}_{4}$ is pulled back to $\mathrm{P}_{4}^{\prime}$ where $\mathrm{P}_{4}^{\prime}$ contacts with the triangular element colliding with the tetrahedron. When more than one triangular element collide with the tetrahedron, $\mathrm{P}_{4}$ ' is calculated for each collision. Among those pulled back points, $\mathrm{P}_{4}$ 's, the point closest to $\mathrm{P}_{3}$ is adopted as the position where $P_{3}$ is moved.

\subsection{Force through Contact Point}

The algorithm of the simulation is shown in Fig. 9 . In this simulation, mechanical interaction of two parts, part 1 and part 2 is considered. The force acts on part 1 from part 2 through each contact point is treated as external force for the iterative calculation of minimizing total energy. The force acts on part 2 


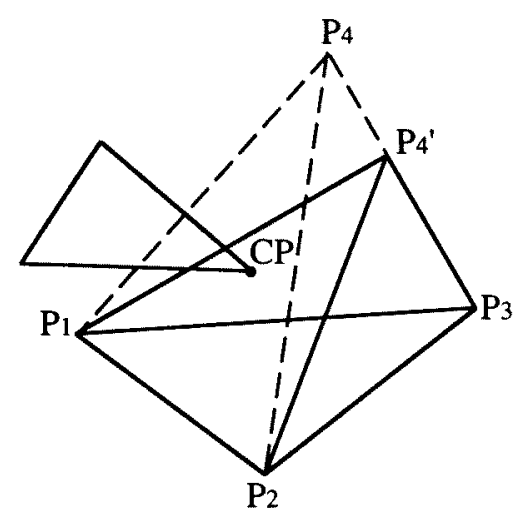

Collision mode 1 : apex to triangle

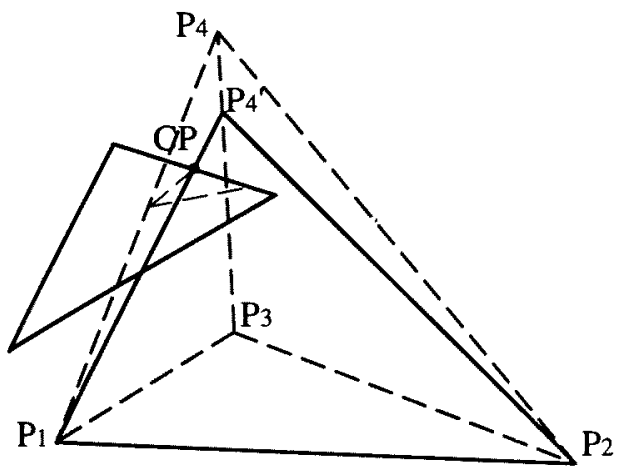

Collision mode 2 : side to side

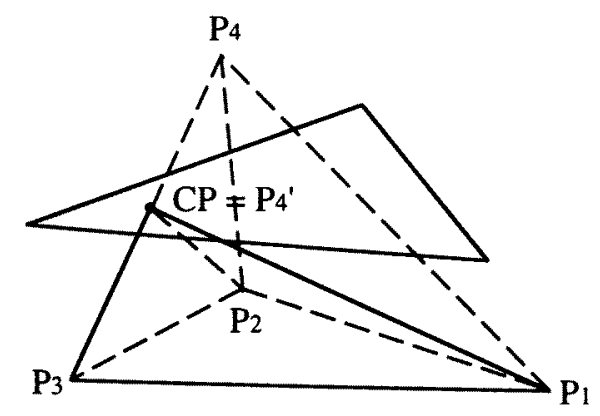

\section{Collision mode 3 : triangle to apex}

Fig. 8 Collision modes.

from part 1 is treated in the same way. The effect of friction is not considered here. In the first step, the force acts on the each apex of two parts is calculated from internal force of the each part. At the same time, each force acts on part 1 from part 2 through the contact point is calculated if the contacts are already detected.

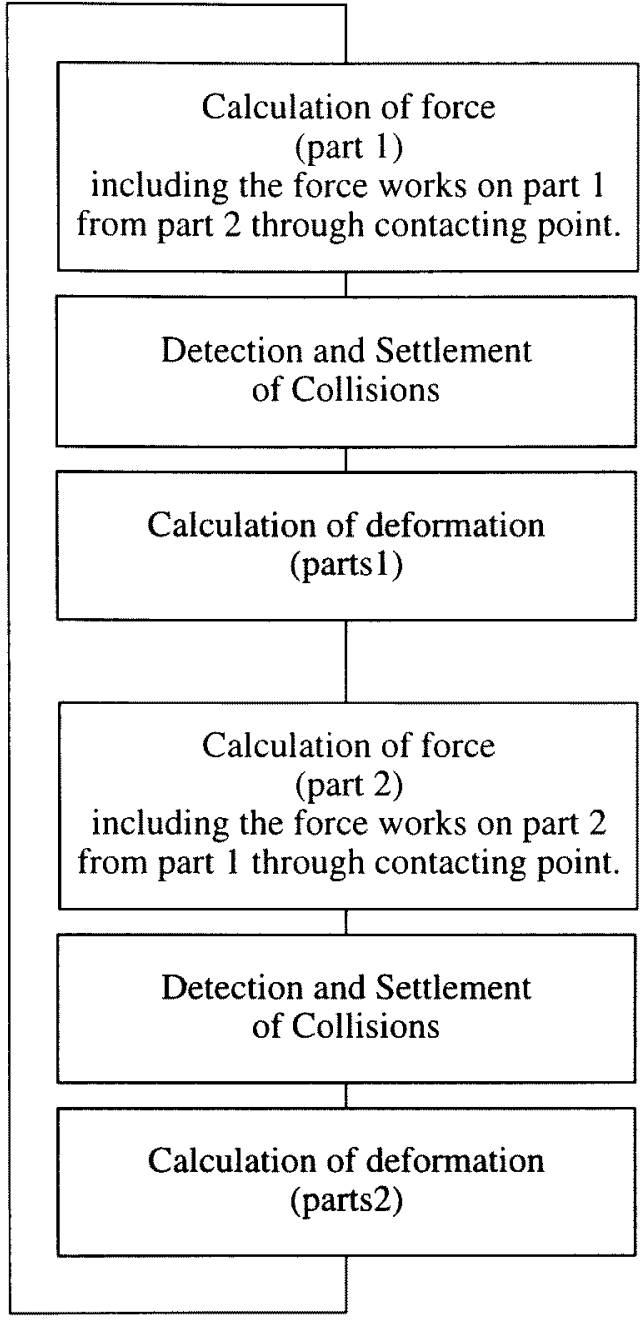

Fig. 9 Algorithm for energy calculation and collision detection.

In the second step, displacement of each apex of part 1 is calculated from the force acts on the apex. In the third step, collisions of part 1 and part 2 are detected and settled to contact with. In the next step, the roles of partl and part 2 are exchanged and the same processes are executed.

When two parts contact with each other, mechanical force acts from one part to another through contact point. The forces act on part 2 from part 1 should be considered for the calculation of the deformation of part 2. In the simulation, forces are considered to act only on apexes. The force from part 1 to part 2 is calculated such that the internal force of part 1 is transmitted to part 2 through the contact point. The 
force of the contact point is calculated from the force of part 1 apexes according to the way of contact described below. For the calculation af part 2 , the force acts on the contact point should be distributed to the part 2 apexes of the triangular element including the contact point. There are three ways that two parts contact with as shown in Fig.8. Those are (1) the contact of an apex of part 1 with a triangular element of part 2, (2) the contact of a side of part 1 with a side of part 2, and (3) the contact of a triangular element of part 1 with an apex of part 2 . In case of (1), the force acts on the contact point is equal to that acts on the apex of part 1 . The force should be distributed into the forces act on the three apexes of the triangular element of part 2. The way of the distribution is determined with the force and 'momentum balance. In case of (2), the force acts on the contact point is the sum of the forces work on the apexes at both edges of the part 1 side. The force of the contact point should be distributed into the apexes at both edges of the side of part 2 in the same way as (1). In case of (3), the force of the contact point is the sum of the forces act on the three apexes of the triangular element of part 1 . The force acts on the apex of part 2 is the same as the force acts on the contact point. The internal force of part 2 and the interaction with part 1 are summed up for each apex of part 2. Deformation of part 2 can be calculated from the force acts on each apex.

\section{Results and Discussion}

With the method above, the seam pucker simulation was improved. The seam pucker simulation test with two strips of fabrics was started with the condition that two strips of virtual fabrics were a little bit displaced from flat and did not contact with each other besides seam line as Fig. 10. In this test, the geometrical method was adopted for the management of collisions to confirm that the program of the geometrical method works properly. Fig.11 is the result of the simulation and the upper fabric is shown in wire frame for display. This figure shows that all the collisions were settled and the two fabrics contacted in several points.

The method was applied to another example of an elastic body wrapped with a belt of fabric. The structure of the elastic body is wire frame as shown in Fig.12. Every side of the body is a virtual spring. The force of the spring is proportional to the displacement and connected springs have stiffness which tries to maintain the angle between the connected two springs. Fig. 13 shows the initial condition of the simulation. The dotted line is the elastic body of original size. In the initial condition, the elastic body was made shrink and located within the belt of fabric. Fig.14 shows the appearance of initial state of the simulation. Fig.15 shows the result of the simulation that the elastic body expanded and contacted with the fabric.
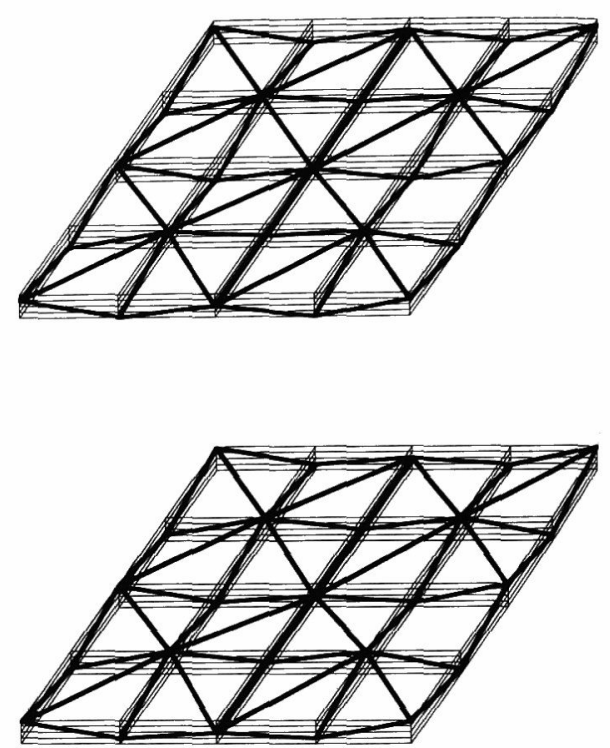

Fig.10 The initial condition of seam pucker simulation with two strips of fabric.

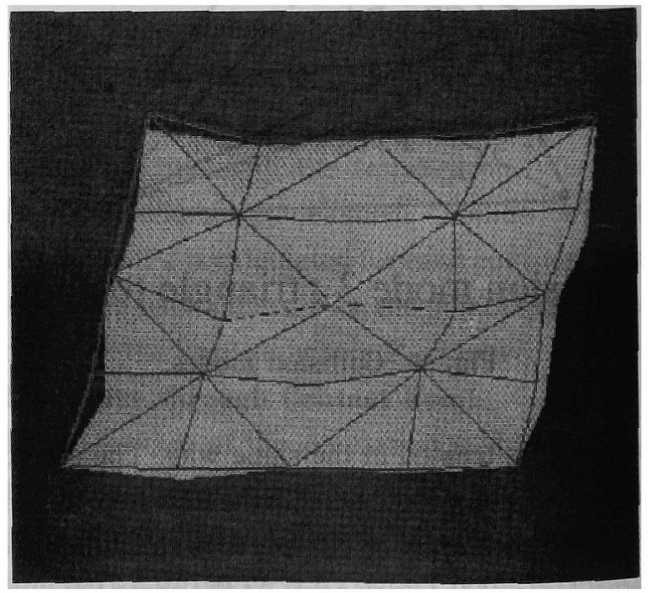

Fig.11 The result of seam pucker simulation with two strips of fabric. 
It is mentioned above that collision detection consists of two processes, global and local. For the local search, "selection" was adopted here. The programming is much simplified by the "selection" because collision detection and management can be coded with the calculation of the planes that make a tetrahedron and the function call of "selection". For the global search, an efficient self collision detection technique was proposed[4]. The technique can be used for the global search and it is suitable for collision detection of deformable objects like fabric. For collision management, some techniques for deformable objects

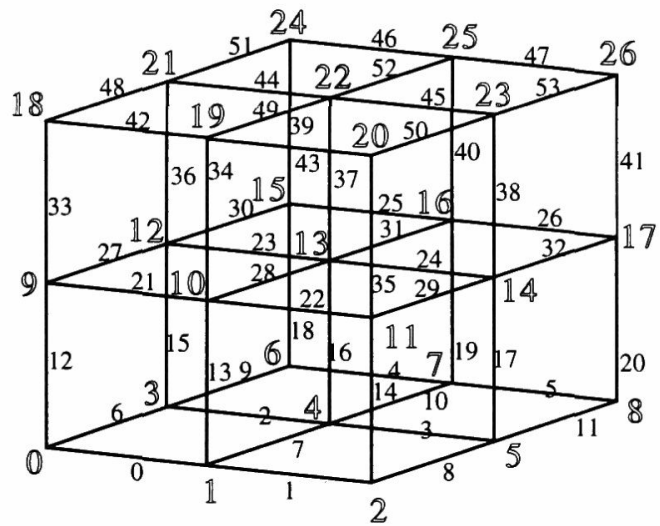

Fig.12 Model for elastic cube. Outline font numbers represent nodes and normal font numbers represent sides.

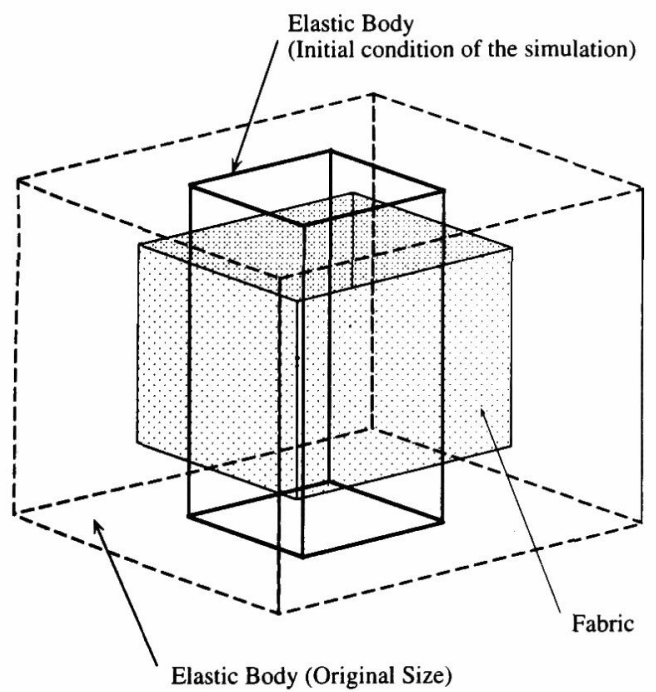

Fig.13 The initial condition of the simulation of an elastic body and fabric.

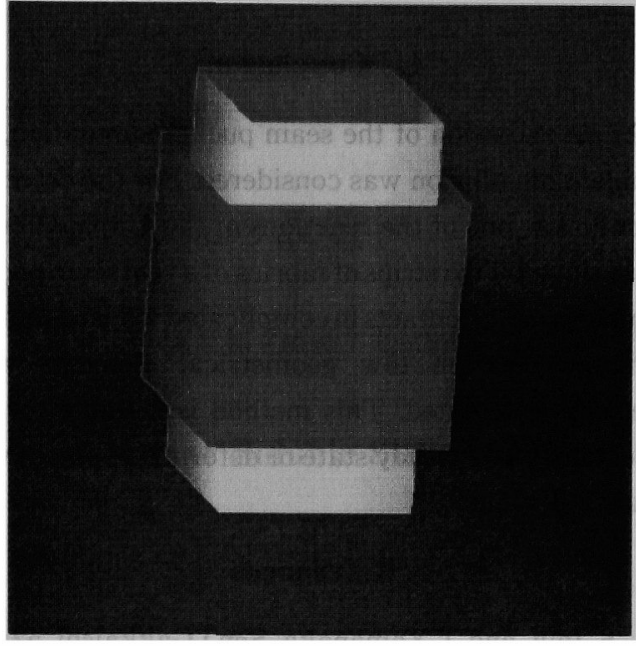

Fig.14 The initial state of the simulation of an elastic body and fabric.

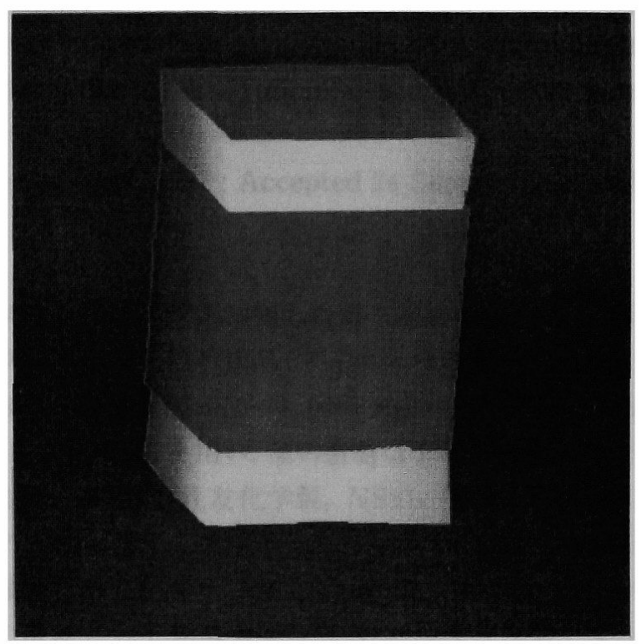

Fig.15 The steady state of the simulation of an elastic body and fabric.

were proposed [5-7]. Those techniques were used for dynamic system in solving the equation of motion. The position and the velocity of an apex were modified with the technique if the apex was going to collide. We have utilized static simulation in which shape of fabric is determined as the minimal energy state because our simulation converges more rapidly than dynamic one. In our simulation, velocity is not considered and movement of the apex does not correspond to the real one. To manage collisions in our simulation, the contact force is calculated instead of position and velocity modification. 


\section{Conclusion}

As an extension of the seam pucker simulation, the problem of collision was considered. For the detection of collision, one of the functions of the graphic library was utilized. Two strips of fabrics of a real seam pucker sample usually contacts in complicated ways so that the contacts between tow geometrical elements were precisely calculated. This method is suitable for the calculation of a steady state of deformable object with many collisions.

\section{References}

1. S. Inui, and T Yamanaka, Sen'i Gakkaishi, 52, 671
(1996).

2. H. Okabe, H. Imaoka, T. Tomiha, and H. Niwaya, Computer Graphics, 26[2], 105 (1992).

3. M. Woo, J. Neider, and T. Davis "OpenGL Programming Guide, Second Edition", Addison Wesley p470 (1997).

4. P. Volino, and N. M. Thalmann, Computer Graphics Forum (EuroGraphics Proc.), 13, 155 (1994).

5. M. Matthew, and W. Jane, Computer Graphics, 22 [4], 289 (1988).

6. B. Herzen, A. Barr, and H. Zatz, Computer Graphics, 24[4], 39(1990).

7. M. Carignan, Y. Yang, N. M. Thalmann, and D. Thalmann, Computer Graphics, 26[2], 99 (1992). 